\title{
Anomalous low temperature stair like coercivity decrease due to magnetostatic coupling between superconducting and ferromagnetic particles in mixed powders
}

\author{
P. Marín, ${ }^{1}$ A. M. Aragón, ${ }^{1}$ M. Lieblich, ${ }^{2}$ A. García Escorial, ${ }^{2}$ P. Crespo, ${ }^{1}$ and A. Hernando ${ }^{1}$ \\ ${ }_{1}^{1}$ Instituto de Magnetismo Aplicado, UCM-ADIF-CSIC, P.O. Box 155 las Rozas, Madrid 28230, Spain \\ ${ }^{2}$ Centro Nacional de Investigaciones Metalúrgicas, CENIM-CSIC, Gregorio del Amo 8, Madrid 28040, Spain
}

(Received 23 April 2012; accepted 1 June 2012; published online 9 July 2012)

\begin{abstract}
Magnetization curves of mixed $\mathrm{Nb}$ and FeSi based micrometric particles have been analyzed. The influence of the dispersion of $\mathrm{Nb}$ particles on the mixture remanence and coercivity has been studied above and below the $\mathrm{Nb}$ superconducting critical temperature. The hysteresis loop shows, at $5 \mathrm{~K}$ and low applied fields, a decrease of both remanence and coercivity with respect to the one of pure ferromagnetic powders as well as a stair like profile. These features are explained as a consequence of the diamagnetic hysteresis loop of $\mathrm{Nb}$ giving rise to local stray fields acting on the ferromagnetic particles at its nearest neighboring. (C) 2012 American Institute of Physics. [http://dx.doi.org/10.1063/1.4733558]
\end{abstract}

\section{INTRODUCTION}

Ferromagnetic powders with adjustable shape and size can be utilized for different applications, e.g., flux enhancement at high frequencies. However, decreasing the sample dimensions leads to an intrinsic magnetic hardening ${ }^{1}$ that can be ascribed to (i) a random anisotropy effect, (ii) the introduction of stresses and defects associated with the usual preparation procedure, and (iii) the unavoidable influence of the inter-particle magnetostatic interactions, ${ }^{2}$ that play an important role in patterned structures for magnetic recording media influencing the switching field distribution and limiting the device density. ${ }^{3}$

With respect to soft ferromagnetic materials, it was observed that outstanding soft material ${ }^{4-6}$ was obtained by $\mathrm{Fe}_{3} \mathrm{Si}$ nanocrystals dispersed in an amorphous phase obtained by the partial devitrification of $\mathrm{Fe}_{73.5} \mathrm{Cu}_{1} \mathrm{Nb}_{3} \mathrm{Si}_{13.5} \mathrm{~B}_{9}$ alloy, which yielded coercivities as low as a few mOe. However, the same alloy when prepared as powders exhibits coercivities of 160 Oe after $60 \mathrm{~h}$ ball milling. ${ }^{7}$

A possible way of controlling the magnetization curve is by mixing powders with different properties such as ferromagnetic-antiferromagnetic ${ }^{8}$ or soft-hard ferromagnetic particles. $^{2}$ The hysteresis behaviour of multiphase nano or micro-structured systems depends first on the morphology and intrinsic properties of each one of the phases, but, in addition, depends crucially on the magnitude and characteristics of the exchange ${ }^{4,6}$ and magnetostatic interactions. ${ }^{2}$

Here we report the magnetic response of a mixture of a $75 \%$ mass (by weight) ferromagnetic $\mathrm{Fe}_{73.5} \mathrm{Cu}_{1} \mathrm{Nb}_{3} \mathrm{Si}_{13.5} \mathrm{~B}_{9}$, (FeSi) powder, obtained by atomization, with $25 \%$ mass $\mathrm{Nb}$ powder; the latter is a type II superconductor at temperatures below $9 \mathrm{~K}\left(\mathrm{~T}_{\mathrm{c}}\right)$. Since the magnetic behaviour of the Nb particles drastically changes on increasing temperature above $\mathrm{T}_{\mathrm{c}}$, the magnetostatic interactions also must be modified and consequently their influence on magnetization curve can be probed through changes of the hysteresis loop below and above $\mathrm{T}_{\mathrm{c}}$. A noticeable reduction of the remanence and coercivity as well as a stair-like shape of the hysteresis loop is observed for temperatures below $\mathrm{T}_{\mathrm{c}}$, whereas the influence is almost negligible above this temperature. The results are discussed in terms of the magnetostatic interactions between both phases by using stray field fluctuations considerations.

\section{EXPERIMENT}

$\mathrm{Fe}_{73.5} \mathrm{Cu}_{1} \mathrm{Nb}_{3} \mathrm{Si}_{13.5} \mathrm{~B}_{9}$ (atomic percent) starting material was atomized in a confined nozzle atomizer ${ }^{9}$ where the liquid melt solidifies rapidly at high cooling rate $\left(10^{3}-10^{5} \mathrm{~K} \mathrm{~s}^{-1}\right)$. The resulting powder was allowed to cool in the inert gas of the atomizer. It was later sieved to achieve separation into different size ranges. In this work, the small, $\sim 25 \mu \mathrm{m}$ size, particles were been used after a $1 \mathrm{~h} 700^{\circ} \mathrm{C}$ annealing. The annealing process leads to phase stabilization as published elsewhere. ${ }^{9} \mathrm{Nb}$ powders, $99.8 \%$ purity, from Sigma Aldrich and $300 \mu \mathrm{m}$ average size, were mixed with the $25 \mu \mathrm{m} \mathrm{FeSi}$ powders and dispersed by vibration forming a mixture $25 \%$ $\mathrm{Nb}$ and $75 \%$ FeSi expressed in mass percent.

Magnetization was measured using VSM-PPMS 6000 Quantum Design magnetometer. The sample studied was prepared by pressing the mixture to form a cylinder $0.75 \mathrm{~mm}$ height and $2.7 \mathrm{~mm}$ diameter. The field is applied along the cylinder axis or z-axis.

\section{RESULTS AND DISCUSSION}

Figure 1 illustrates the hysteresis loop of the $\mathrm{Nb}$ powders, measured at $5 \mathrm{~K}$, that is characteristic of type II superconductors. The Nb coercive field is close to $200 \mathrm{Oe}$. Both descendent branches of the hysteresis loop show a maximum of magnetization, $M=5.8 \mathrm{emu} / \mathrm{g}$ for applied fields of 400 Oe. The remanent magnetization is $1.2 \mathrm{emu} / \mathrm{g}$. Figure 2 depicts the saturation hysteresis loops obtained for pure FeSi powders, for the $25 \% \mathrm{Nb}-75 \% \mathrm{FeSi}$ mixture and for pure $\mathrm{Nb}$ at $5 \mathrm{~K}$ (Fig. 2(a)) and $300 \mathrm{~K}$ (Fig. 2(b)). The low applied field region of both curves is shown in detail in Figures 3 and 4 where the $\mathrm{Nb}$ contribution has been omitted. 


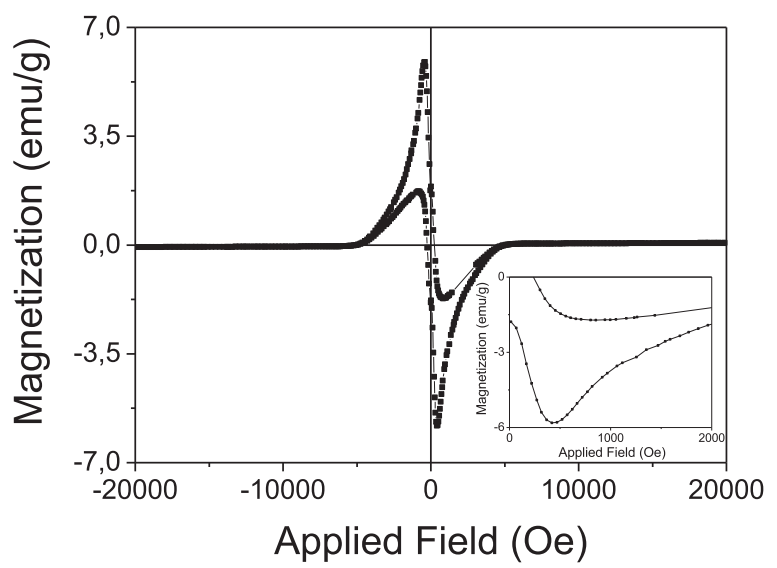

FIG. 1. Hysteresis loop for Nb powders, measured at $5 \mathrm{~K}$. The inset shows a detail for the first branch.

Figures 2(a) and 2(b) indicate that at $\mathrm{T}=5 \mathrm{~K}$ as well as at $\mathrm{T}=300 \mathrm{~K}$, the saturation magnetization of the mixture is approximately 0.75 times the saturation magnetization of the pure FeSi powders. Note that at $\mathrm{T}=5 \mathrm{~K}$ and under the maximum applied field of $50 \mathrm{kOe}$, the $\mathrm{Nb}$ particles are paramagnetic. Therefore, the saturation magnetization relation experimentally observed between those of the mixture and the pure FeSi phases is in agreement with the mass percentage of the mixture, when the paramagnetic contribution of the $\mathrm{Nb}$ phase is neglected. It is important to note that from the shape of the curves shown in Figures 2(a) and 2(b) it
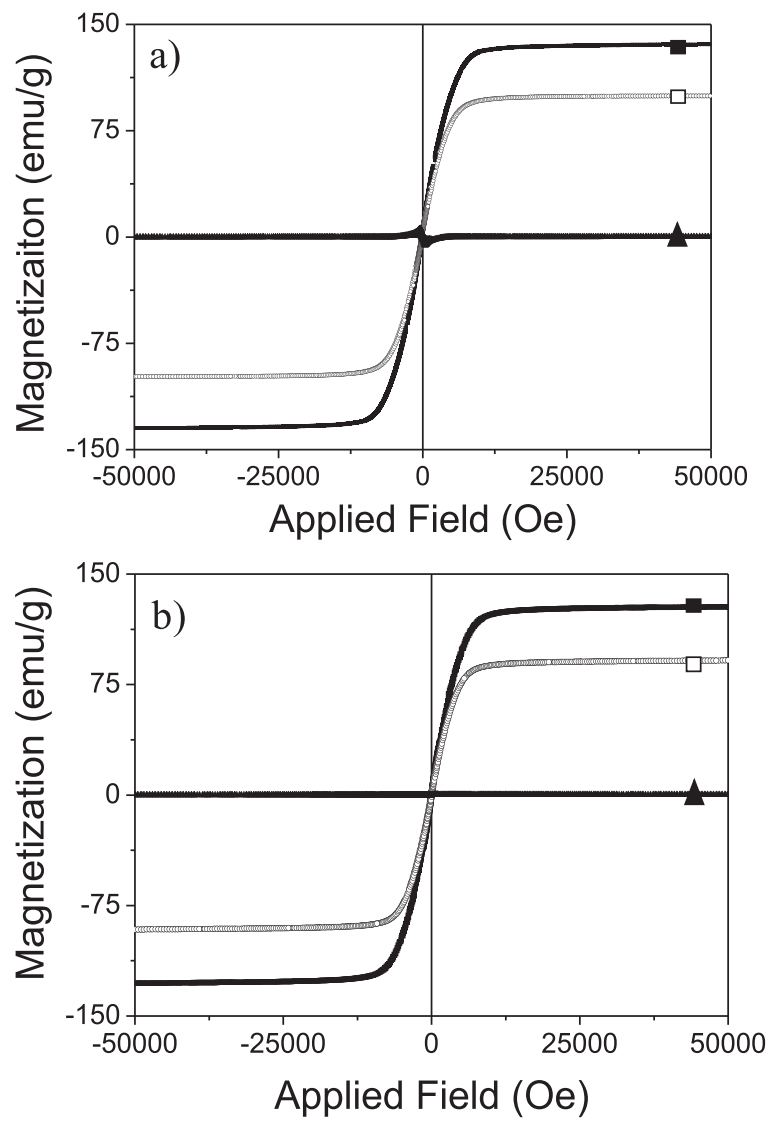

FIG. 2. Saturation magnetization hysteresis loops for pure FeSi ( $\mathbf{\square})$, pure $\mathrm{Nb}(\boldsymbol{\Delta})$, and for the mixture $25 \% \mathrm{Nb}-75 \% \mathrm{FeSi}(\bigcirc)$ measured at $5 \mathrm{~K}$ (a) and at $300 \mathrm{~K}(\mathrm{~b})$.

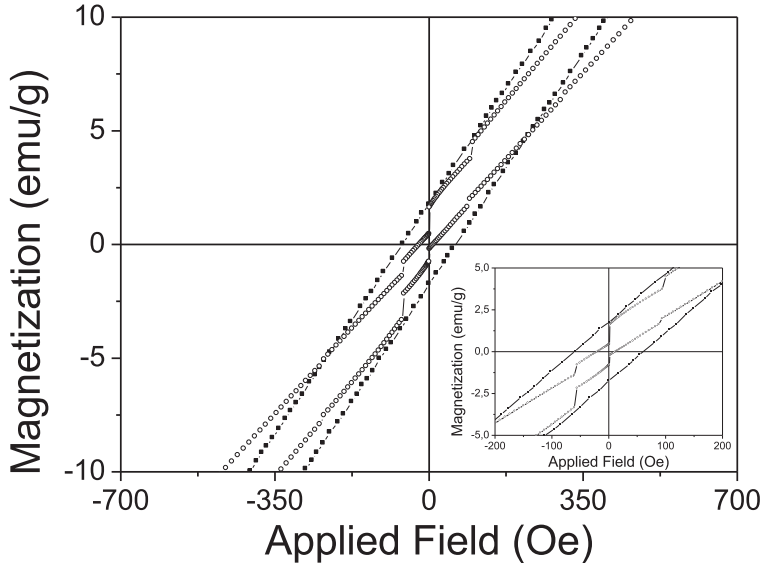

FIG. 3. Detail of the hysteresis loops obtained at $5 \mathrm{~K}$ for pure $\mathrm{FeSi}$ powders and for the mixture $25 \% \mathrm{Nb}-75 \% \mathrm{FeSi}(\mathrm{O})$.

turns out that long range magnetostatic interactions govern the magnetization curves through the macroscopic demagnetizing factor of the sample, $N$. The size of the FeSi particles is well above the single domain range and consequently magnetizes by nucleation and propagation of domains.

As shown in Figure 3, the coercivity of the mixture at $5 \mathrm{~K}$ is $15 \mathrm{Oe}$, lower than that of pure FeSi powders that reaches $60 \mathrm{Oe}$. The remanence of the mixture, $0.60 \mathrm{emu} / \mathrm{g}$ is less than one half that the measured for the pure FeSi phase, $1.80 \mathrm{emu} / \mathrm{g}$. Hence, the hysteresis loop of the mixture undergoes a noticeable narrowing at low fields (between 150 and $-150 \mathrm{Oe}$ ) respect to that of the pure FeSi phase. The effect of $\mathrm{Nb}$ powders, for temperatures above its critical temperature, seems to be opposite and exhibits a trend to increase both remanence and coercivity (Figure 4). This last observation coincides with the expected effect produced by the introduction of non magnetic inclusion in a ferromagnetic system that necessarily leads to a shortening of the exchange correlation length and subsequent hardening. ${ }^{6}$

A second and intriguing characteristic of the hysteresis loop of the mixture, measured at $5 \mathrm{~K}$, is the stair-like profile of both hysteresis branches that can be seen in Figure 3. This type of magnetization curves is also characteristic of other

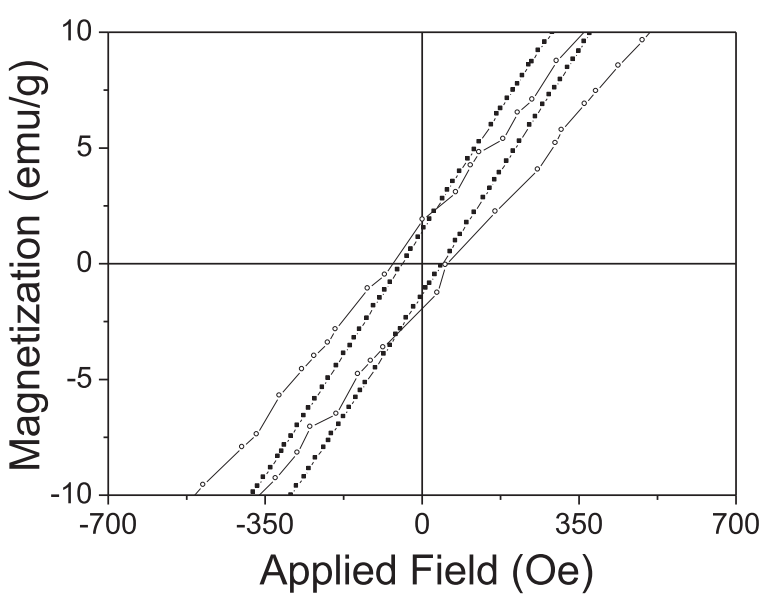

FIG. 4. Detail of the hysteresis loops obtained at $300 \mathrm{~K}$ for pure FeSi powders and for the mixture $25 \% \mathrm{Nb}-75 \% \mathrm{FeSi}(\mathrm{O})$. 
very different magnetic systems as for example antiferromagnetic structures with a dispersion of metamagnetic field transitions and systems exhibiting magnetic avalanches. ${ }^{10-14}$ However, stair-like hysteresis profiles have been found and reported to be originated generally by magnetostatic interactions. ${ }^{15}$

Since the narrowing in the hysteresis loop as well as the stair-like behaviour disappears at $300 \mathrm{~K}$, it turns out that both effects must likely be associated with the presence of the superconducting $\mathrm{Nb}$ particles. Therefore, they must be explained in terms of the diamagnetism of the superconducting particles that modify the macroscopic magnetization and gives rise to peculiar discrete magnetostatic interactions between first neighbours of the two different phases. ${ }^{2,15}$

Let us start by estimating the remanence and coercivity decrease when magnetostatic interactions between both phases are neglected. From the measured remanence of the phases determined separately, one might conclude that the magnetization of $\mathrm{FeSi}$ and $\mathrm{Nb}$ particles are oriented along opposite directions in the remanent state; after considering the respective mass percentage, the expected mixture remanence is found to be $1 \mathrm{emu} / \mathrm{g}$. However, the measured remanence of the experimental mixture is $0.6 \mathrm{emu} / \mathrm{g}$. When the applied field is reversed to negative values, the slope of the descendent branch of the hysteresis of the pure FeSi powders in the vicinity of the coercive field is $3 \times 10^{-2} \mathrm{emu} / \mathrm{g}$ Oe (Figure 3). The magnetization of the $\mathrm{Nb}$ phase is, according to Figure 1, smaller than the remanence, $1.1 \mathrm{emu} / \mathrm{g}$. Considering $0.25 \%$ mass percentage of $\mathrm{Nb}$ in the mixture, this $1.1 \mathrm{emu} / \mathrm{g}$ leads to a value of $0.27 \mathrm{emu} / \mathrm{g}$. Consequently, the expected decrease of the coercivity due to the $\mathrm{Nb}$ negative remanent contribution becomes $10 \mathrm{Oe}$, which would decrease the coercivity of pure $\mathrm{FeSi}$ down to $50 \mathrm{Oe}$, whereas the value experimentally determined is $15 \mathrm{Oe}$. It can be concluded that even though the diamagnetic contribution of the $\mathrm{Nb}$ superconducting particles accounts for the qualitative changes observed in the hysteresis loop the quantitative experimental observations require further insight to be satisfactorily explained.

Let us estimate the discrete, short range magnetostatic interactions induced by oppositely aligned $\mathrm{Nb}$ particles and surrounding $\mathrm{FeSi}$ particles. By considering the densities of $\mathrm{FeSi}, \rho_{\mathrm{FeSi}}$, to be approximately $7 \mathrm{~g} / \mathrm{cm}^{3}$ and of $\mathrm{Nb}$, $\rho_{\mathrm{Nb}}=8.14 \mathrm{~g} / \mathrm{cm}^{3}$, it can be found that the number of $25 \mu \mathrm{m}$ FeSi particles in the mixture per cubic centimetre is approximately $10^{6}$, whereas the number of $300 \mu \mathrm{m} \mathrm{Nb}$ particles should be close to $10^{3}$. Thus, the percentage of the total number of FeSi particles located at the first neighbourhood of all the $\mathrm{Nb}$ particles per unit volume becomes $10 \%$ for maximum packing factor. A more realistic estimation of the average yields $5 \%$ of $\mathrm{FeSi}$ particles that are actually located within the nearest neighbours shell.

All FeSi particles located outside the nearest neighbour locations of $\mathrm{Nb}$ particles, that are more than $90 \%$ of the total number of $\mathrm{FeSi}$ particles per unit volume, form the matrix. According to the curves shown in Figures 2 and 4, the magnetization process of the matrix is not noticeably modified, at least in a first order, by the presence of the $\mathrm{Nb}$ particles. For the sake of simplicity, it will be assumed that, according to Figures 2 and 3, the matrix magnetization, $M_{m}$, can be roughly expressed in emu/g as $M_{m}=\left(H_{a p}\right)$ $\left.4 \pi N \rho_{F e S i}\right)=3 \times 10^{-2} H_{a p}$. As concerns the short range magnetostatic interactions, three different phases can be distinguished: the $\mathrm{FeSi}$ matrix, the $\mathrm{FeSi}$ interface formed by those particles located at the shell of nearest neighbouring of $\mathrm{Nb}$ particles, and the $\mathrm{Nb}$ phase. The magnetic field acting on the $\mathrm{FeSi}$ particles forming the interface is the sum of the fields produced by the spherical matrix-interface plus the $\mathrm{Nb}$ particle, sum that can be approximated as

$$
\begin{aligned}
H_{\text {shell }} & =\frac{4 \pi \rho_{F e S i} M_{m}}{3}+\frac{4 \pi \rho_{N b} M_{N b} R^{3}}{3(R+r)^{3}}\left(3 \cos ^{2} \theta-1\right) \\
& =27.7 M_{m}+25.4 M_{N b}\left(3 \cos ^{2} \theta-1\right)
\end{aligned}
$$

where $\mathrm{R}$ and $\mathrm{r}$ hold for the $\mathrm{Nb}$ and FeSi particle radii, respectively, and $\theta$ is the angle formed by the position vector of the $\mathrm{FeSi}$ particle with origin at the centre of the $\mathrm{Nb}$ particle and the $\mathrm{z}$ axis.

Figure 5(a) indicates the magnetization of $\mathrm{Nb}$ and $\mathrm{FeSi}$ particles in the descendent branch of the hysteresis loop. As illustrated by Figure 5(a), the interface FeSi particles can be classified in three groups according to its angular position respect to the $\mathrm{Nb}$ central particle: (a) polar particles over which the field produced by the $\mathrm{Nb}$ magnetization is opposite to its magnetization, (b) equatorial particles over which the field is parallel to its magnetization, and (c) neutral particles located at angles $\theta$ for which $3 \cos ^{2} \theta$ is close to 1 . Figure $5(\mathrm{~b})$ shows the component $\mathrm{z}$, parallel to the applied field, of the stray fields produced by the $\mathrm{Nb}$ particle at the first shell.

The field acting on the $\mathrm{Nb}$ particles that is given by the sum of the field produced by the matrix-shell spherical interface plus the field produced by the $n$ FeSi particles contained at the first shell is

$$
H_{N b}=4 \pi \rho_{F e S i}\left[\frac{M_{m}}{3}+\sum_{i} \frac{M_{\text {shell }} r^{3}}{3(R+r)^{3}}\left(3 \cos ^{2} \theta_{i}-1\right)\right],
$$

$M_{\text {shell }}$ is the magnetization of the FeSi particles of the first shell that is determined by $H_{\text {shell }}$ therefore through relation (1), by $M_{m}$ and $M_{N b}$. But $M_{N b}$ depends on $H_{N b}$ and therefore, according to relation (2), on $M_{m}$ and $M_{\text {shell }}$. Whereas $M_{m}$ can be approximated by the expression $M_{m}=3 \times 10^{-2} H_{a p}$, the values of $M_{N b}$ and $M_{\text {shell }}$ cannot be generally expressed as a function of $H_{a p}$.

According to relation (1), $H_{\text {shell }}$ will be influenced by $M_{N b}$ only for values of the magnetization matrix close to the magnetization of the $\mathrm{Nb}$ particles. Since the maximum magnetization of $\mathrm{Nb}$ is $5.8 \mathrm{emu} / \mathrm{g}$, the interaction effects are only relevant in the range for which $M_{m}$ varies between 6 and -6 $\mathrm{emu} / \mathrm{g}$, that corresponds to applied fields in the interval between 250 and -250 Oe. ${ }^{16}$ Within this range of applied fields, $H_{\text {shell }}$ can be oriented either along the plus or the minus directions depending on the angular position of the FeSi particles in the shell.

Along the descendent branch of the hysteresis loop and in the range for which $M_{m}$ and $M_{N b}$ are oriented along 


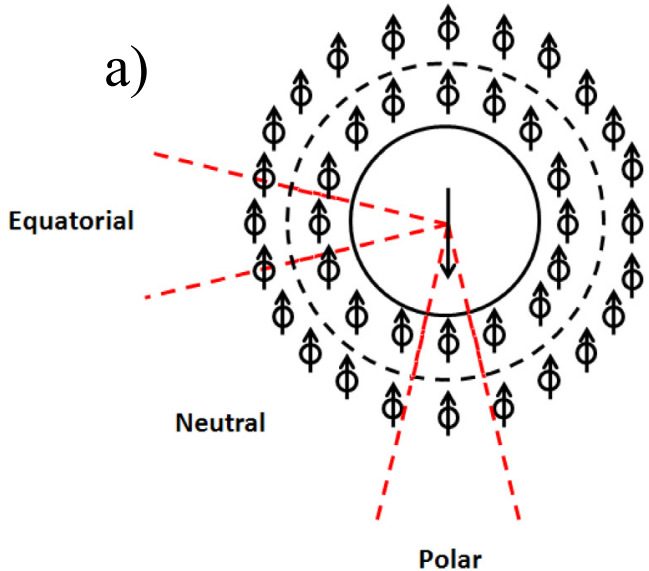

opposite directions, relation (1), shows that at the polar positions $H_{\text {shell }}$ vanishes for $M_{m}=1.8 M_{N b}$. The coercive field of the shell particles, achieved for $H_{\text {shell }}=-60 \mathrm{Oe}$, can correspond to positive applied fields, since according to Eq. (1) this condition becomes

$$
-60=27.7 M_{m}-50.8 M_{N b}\left(3 \cos ^{2} \theta-1\right)=(27.7-50.8 \alpha) M_{m},
$$

where $\alpha=M_{N b} / M_{m}$.

Note that if $\alpha>0.54$ the coefficient of $M_{m}$ in relation (3) is negative and then is possible with positive $H_{a p}$ to obtain negative $H_{\text {shell }}$. According to the curve shown in Figures 3, the remanence of $M_{m}$ is $1.8 \mathrm{emu} / \mathrm{g}$ and gives rise, according to relation (2) and neglecting its second term, to $H_{N b}$ close to $60 \mathrm{Oe}$, that corresponds to a negative $M_{N b}=2 \mathrm{emu} / \mathrm{g}$ (Fig. 1). Therefore, close to the region of zero applied field, $\alpha$ takes values above 1 , thereby higher than 0.6. Hence, FeSi particles located at the polar regions of the first shell can switch the magnetization even under positive applied fields due to the magnetic field produced by the $\mathrm{Nb}$ particles. When the magnetization of a polar particle reverses the magnetostatic coupling between the FeSi particles forming the first shell it can give rise to the stair-like jumps between metastable states as experimentally observed and theoretically analysed in Ref. 15. For those applied field for which some particles of neutral ( $H_{a p}$ close to zero) and equatorial particles
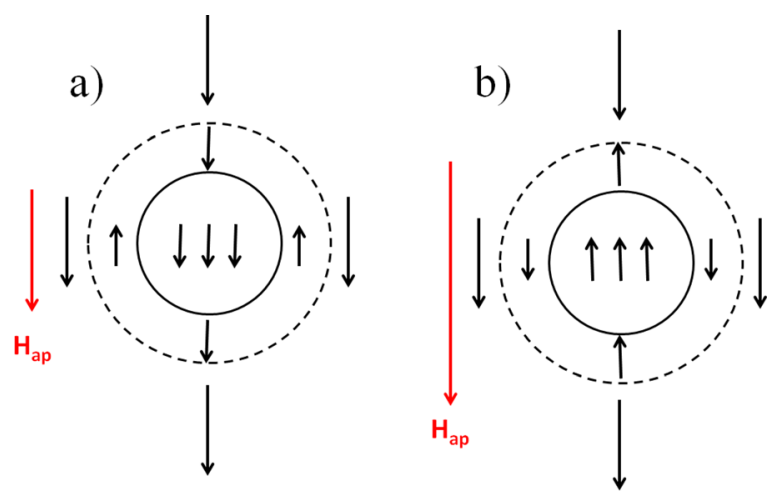

FIG. 6. The reversal of the $\mathrm{Nb}$ magnetization from negative to positive inducing the immediate reversal of the equatorial particles from positive to negative.
( $H_{a p}$ negative) reverse the same type of jumps are expected to be induced.

Note that, as shown in Figure 3, the strength of the magnetization jump steps (2.75 and $2.25 \mathrm{emu} / \mathrm{g}$ at the descendent and ascendant branches, respectively) roughly represents $2 \%$ of the total magnetization switch which is the order of either the total switch of the $\mathrm{Nb}$ phase magnetization or the switch of a fraction of the FeSi particles located at the first neighbouring positions of the $\mathrm{Nb}$ particles.

Following the descendent branch of the hysteresis loop is interesting to remark the effect produced on $H_{N b}$ by the reversal of the applied field to negative values. In this situation $M_{m}$ is negative and the second term in Eq. (2) is also negative as the polar particles had eventually reversed the magnetization at positive applied fields (Figure 6). When the sum of both terms in relation (2) reaches the coercivity shown in Figure 1, that is $200 \mathrm{Oe}$, the magnetization of $\mathrm{Nb}$ vanishes. If the second term in Eq. (2) is neglected ${ }^{17}$ the reversal of $\mathrm{Nb}$ magnetization would take place for $M_{m}=6 \mathrm{emu} / \mathrm{g}$. However, when the second term is taken into account the reversal could take place at negative applied fields for which $M_{m}$ could be as low as $2.5 \mathrm{emu} / \mathrm{g}$. The reversal of the $\mathrm{Nb}$ magnetization from negative to positive would immediately induce the reversal of the equatorial particles from positive to negative, as illustrated in Figure 6.

\section{CONCLUSIONS}

In conclusion, the dispersion of superconducting particles in a ferromagnetic powder gives rise to a narrowing of the hysteresis loop through the decrease of both remanence and coercivity. This perturbation of the magnetization process is only observed under applied fields for which the magnetization of both phases is similar. The diamagnetic hysteresis loop of the superconducting phase accounts for the qualitative behaviour. However, the explanation of the stair like profile of the magnetization curve as well as the quantitative changes measured in remanence and coercivity require magnetostatic coupling considerations. The analysis carried out in this report shows that the coupling only affects to a small fraction, $2 \%$, of FeSi particles forming the interface between the FeSi matrix and the $\mathrm{Nb}$ inclusions. By increasing the temperature of the sample above the critical superconducting temperature, the effect of the $\mathrm{Nb}$ becomes 
opposite and a weak increase of both coercivity and remanence with respect to those of pure FeSi phase is appreciated. This hardening is expected when non magnetic inclusions are introduced in a ferromagnetic system. It has been shown that typical characteristics of the ferromagnetic powders hysteresis loop, as are coercivity and remanence, can be controlled at low temperature by mixing with adequate superconducting particles.

\section{ACKNOWLEDGMENTS}

This work has been supported by the MEC of Spain (Consolider-Ingenio 2010 Contract Nos. CSD2007-0010 and MAT2009-14741-C02-01), S2009 MAT 1726, and Project Nos. TSI-020100-2011-280 and IPT-2011-0893-42000.

\footnotetext{
${ }^{1}$ A. Hernando, P. Marín, M. López, T. Kulik, L. K. Varga, and G. Hadjipanayis, Phys. Rev. B 69, 052501 (2004).

${ }^{2}$ A. Hernando, D. X. Chen, M. Pardavi-Horvath, and J. M. González, Phys. Rev. B 63, 052404 (2001).

${ }^{3}$ M. Pardavi-Horvath, IEEE Trans. Magn. 32, 4458 (1996). The influence of magnetostatic interactions on the hysteresis of a system of particles is analyzed by P. Allia, M. Coisson, M. Knobel, P. Tiberto, and F. Vinai, Phys. Rev. B 60, 12207 (1999).
}

${ }^{4}$ G. Herzer, IEEE Trans. Magn. 25, 332 (1989); 26, 1397 (1990); Scr. Metall. Mater. 33, 1741 (1995).

${ }^{5}$ J. F. Loffler, H. B. Braun, and W. Wagner, Phys. Rev. Lett. 85, 1990 (2000).

${ }^{6}$ A. Hernando and T. Kulik, Phys. Rev. B 49, 7064 (1994).

${ }^{7}$ J. S. Garitaonaindia, L. Righi, J. M. Barandiarán, P. Gorria, F. Leccabue, and B. E. Watts, Mater. Sci. Forum 235-238, 193 (1998).

${ }^{8}$ J. Sort, J. Nogués, X. Amils, S. Suriñach, J. S. Muñoz, and M. Baró, Appl. Phys. Lett. 75(20), 3177 (1999).

${ }^{9}$ A. García-Escorial, M. Lieblich, M. López, and P. Marín, J. Alloys Compd. 509S, S239-S242 (2011).

${ }^{10}$ D. Grundler, G. Meier, K.-B. Brooks, Ch. Heyn, and D. Heitmann, J. Appl. Phys. 85, 6175 (1999); J. M. Gonzalez, O. A. Chubykalo, A. Hernando, and M. Vázquez, J. Appl. Phys. 83, 7393 (1998).

${ }^{11}$ S. Hébert, V. Hardy, A. Maignan, R. Mahendiran, M. Hervieu, C. Martin, and B. Raveau, J. Solid State Chem. 165, 6-11 (2002).

${ }^{12}$ V. Hardy, A. Maignan, S. Hébert, C. Yaicle, C. Martin, M. Hervieu, M. R. Lees, G. Rowlands, D. Mc, K. Paul, and B. Raveau, Phys. Rev. B 68, 220402(R) (2003)

${ }^{13}$ G. Brown, M. A. Novotny, and P. A. Rikvold, J. Appl. Phys. 87, 4792 (2000).

${ }^{14}$ V. V. Ustinov, M. A. Milayev, L. N. Romashev, T. P. Krinitsina, A. M. Burkhanov, V. V. Lauter-Pasyuk, and H. J. Lauter, J. Magn. Magn. Mater. 300, e281-e283 (2006).

${ }^{15}$ A. A. Fraerman and M. V. Sapozhnikov, Phys. Rev. B 65, 184433 (2002).

${ }^{16} \mathrm{It}$ is precisely for this range of applied fields and magnetization when the modification of the hysteresis curve due to the $\mathrm{Nb}$ inclusions is experimentally observed as depicted by Figure 3.

${ }^{17}$ This condition holds for a spherical symmetry distribution of the FeSi particles of the interface around the $\mathrm{Nb}$ particle. 